INPLASY

PROTOCOL

To cite: Zhang et al. Effects of intensive blood pressure control on mortality and cardio-renal function in chronic kidney disease patients. Inplasy protocol 202130001. doi:

10.37766/inplasy2021.3.0001

Received: 01 March 2021

Published: 01 March 2021

Corresponding author:

Yan-Hong Tuo

tuoyanhongh@163.com

Author Affiliation:

The Central Hospital of Wuhan, Tongji Medical College, Huazhong University of Science and Technology

Support: At our own expense.

Review Stage at time of this submission: Data analysis.

Conflicts of interest:

None declared.

\section{Effects of intensive blood pressure control on mortality and cardio-renal function in chronic kidney disease patients}

Zhang, Y1; Li, JJ2; Wang, AJ3; Li, T4; Tuo, YH5.

Review question / Objective: Effects of intensive blood pressure control on mortality and cardio-renal renal function in chronic kidney disease patients.

Condition being studied: Blood pressure (BP) variability is highly correlated with cardiovascular and kidney outcomes in patients with chronic kidney disease (CKD). However, appropriate blood pressure targets in patients with CKD remain uncertain.

Information sources: PubMed, Embase and the Cochrane Library.

INPLASY registration number: This protocol was registered with the International Platform of Registered Systematic Review and Meta-Analysis Protocols (INPLASY) on 01 March 2021 and was last updated on 01 March 2021 (registration number INPLASY202130001).

\section{INTRODUCTION}

Review question / Objective: Effects of intensive blood pressure control on mortality and cardio-renal renal function in chronic kidney disease patients.
Condition being studied: Blood pressure (BP) variability is highly correlated with cardiovascular and kidney outcomes in patients with chronic kidney disease (CKD). However, appropriate blood pressure targets in patients with CKD remain uncertain. 


\section{METHODS}

Participant or population: Chronic kidney disease patients.

Intervention: Intensive blood pressure control.

Comparator: Blood pressure control.

Study designs to be included: RCTs.

Eligibility criteria: (1) Only randomized controlled trials (RCTs) were included in the study. (2) Participants were patients with CKD or included in a subgroup of CKD. (3) Participants were over 18 years of age. (4) One of the following outcomes must have been included: cardiovascular disease death, all-cause mortality, composite cardiovascular events, composite renal outcome, serum creatinine level doubling, $50 \%$ reduction in GFR, or serious adverse events (SAEs). (5) Different antihypertensive targets had to be included in the study. The experimental group was the intensive BP control group or the antihypertensive drug treatment group, and the control group was the standard BP group or the placebo group.

Information sources: PubMed, Embase and the Cochrane Library.

Main outcome(s): Doubling of serum creatinine level or $50 \%$ reduction in GFR, Composite renal outcome,Cardiovascular outcomes,All-cause mortality.

Quality assessment / Risk of bias analysis: Sensitivity analysis was performed to calculate the influence of each RCT by omitting of individual studies. The results showed that no individual studies significantly affected the heterogeneity, which indicates that the results of our statistical analysis are robust. Publication bias was examined using the funnel plot method. Heterogeneity among all the studies was tolerable $(12<50 \%)$. This indicates that the results of our study are relatively reliable.
Strategy of data synthesis: STATA 16.0 (Stata Corp LP, College Station, TX, USA) was used to perform statistical analyses.

Subgroup analysis: Subgroup analysis with sample size and follow-up were conducted in main results.

Sensitivity analysis: Sensitivity analysis was performed to calculate the influence of each RCT by omitting of individual studies. The results showed that no individual studies significantly affected the heterogeneity, which indicates that the results of our statistical analysis are robust.

Country(ies) involved: China.

Keywords: chronic kidney disease, intensive BP control, renal outcomes, cardiovascular outcomes, meta-analysis.

Contributions of each author:

Author 1 - Yong Zhang.

Author 2 - Jingjing Li.

Author 3 - Anjun Wang.

Author 4 - Tian Li.

Author 5 - Yan-Hong Tuo. 